\title{
Obstetric hospital preparedness for a pandemic: an obstetric critical care perspective in response to COVID-19
}

https://doi.org/10.1515/jpm-2020-0281

Received June 18, 2020; accepted June 24, 2020; published online August 3, 2020

Abstract: The Coronavirus disease 2019 (COVID-19) caused by Severe Acute Respiratory Syndrome Coronavirus-2 (SARS-CoV-2) pandemic has had a rapid and deadly onset, spreading quickly throughout the world. Pregnant patients have had high mortality rates, perinatal losses, and Intensive Care Unit (ICU) admissions from acute respiratory syndrome Coronavirus (SARS-CoV) and Middle East respiratory syndrome Coronavirus (MERS-CoV) in the past. Potentially, a surge of patients may require hospitalization and ICU care beyond the capacity of the health care system. This article is to provide institutional guidance on how to prepare an obstetric hospital service for a pandemic, mass casualty, or natural disaster by identifying a care model and resources for a large surge of critically ill pregnant patients over a short time. We recommend a series of protocols, education, and simulation training, with a structured and tiered approach to match the needs for the patients, for hospitals specialized in obstetrics.

Keywords: COVID-19; disaster manual; hospital preparedness; isolation; obstetric; pandemic.

\section{Introduction}

The Coronavirus disease 2019 (COVID-19) caused by Severe Acute Respiratory Syndrome Coronavirus-2 (SARS-CoV-2) pandemic has had a rapid and deadly onset, spreading quickly throughout the world $[1,2]$. The initial disease

\footnotetext{
*Corresponding author: Scott Harvey, MD, MS, University of Hawaii at Manoa, John A Burns School of Medicine, Department of Obstetrics, Gynecology and Women's Health, 1319 Punahou Street, Suite 824, Honolulu, Hawaii 96822, USA. Phone: (+001) 808772 2700, Fax: (+001) 808944 2174, E-mail: sharvey@hawaii.edu Ivica Zalud: University of Hawaii at Manoa, John A Burns School of Medicine, Department of Obstetrics, Gynecology and Women's Health, Honolulu, Hawaii, USA; Kapiolani Medical Center for Women and Children, Department of Obstetrics/Gynecology and Womens Health 1319 Punahou Street Suite 824, Honolulu, Hawaii 96816, USA, E-mail: ivica@hawaii.edu
}

outbreak in Wuhan, China estimated that $81 \%$ percent of patients contracted a mild illness, with 14 and $5 \%$ of patients developing severe and critical illness, respectively [3]. With an overall mortality rate of about $2.3 \%$, there was a $49 \%$ mortality rate in the critically ill cohort [3]. The incidence of disease severity is similar in the United States, where $19 \%$ of patients with COVID-19 infection are hospitalized and 3-6\% requires care in the Intensive Care Unit (ICU), with an overall $1.8-3.4 \%$ mortality rate [4]. Acute respiratory failure is the commonest disease feature that requires ICU treatment and monitoring [5]. From a population perspective, if $20 \%$ of patients who contract the disease will likely require hospitalization, and millions are infected, the capacity at which the health care system can treat this surge of patients may be exceeded. For this reason, many hospital systems worldwide have needed to adapt their strategy for health care delivery to accommodate a large volume of patients in short period of time [6]. The need for more mechanical ventilators, medications, and medical personnel was quickly identified as the hospital resources in some regions became overwhelmed.

Pregnant patients have had a grim history with prior outbreaks from Coronavirus infections. With altered cellmediated immunity, higher oxygen consumption, and limited cardiorespiratory reserve, pregnant women are at higher risk for pneumonia and respiratory failure due to pathogens [7]. Reports of pregnant women infected from acute respiratory syndrome Coronavirus (SARS-CoV) and Middle East respiratory syndrome Coronavirus (MERS-CoV) note an approximate $25 \%$ maternal mortality rate and higher rates of poor perinatal outcomes and ICU admissions [7-10]. To date, a few obstetric patients have contracted COVID-19, so data is lacking on how severe this disease will be on pregnant women [7].

Social distancing, travel restrictions, and public education has effectively reduced COVID-19 transmission and has "flattened the curve" in some regions of the world, including Hawaii [11, 12]. With only a few people getting contracting the disease, these regions will not demonstrate "herd immunity" making us vulnerable to an additional surge when social distancing is relaxed. We have observed the devastation of large surge volumes of patients in other regions, prompting us to develop a comprehensive plan in 
case of a surge of infectious patients occurs in our region characterized by remoteness and isolation as an archipelago. We are a Women and Children's hospital without many of the adult hospital service lines but are part of a larger hospital system that does have typical adult service lines. Our hospital delivers about 6,000 babies per year, has a Level IV Neonatal Intensive Care Unit, and a full myriad of gynecologic services to include gynecology oncology, urogynecology, reproductive endocrinology, family planning, maternal fetal medicine, and obstetric critical care.

The purpose of this article is to provide institutional guidance on how to prepare an obstetric hospital service for a pandemic, mass casualty, or natural disaster by identifying a care model and resources for a large surge of critically ill pregnant patients over a short time. For this reason, it was necessary to develop a disaster plan to care for critically ill obstetric patients, with principles discussed below [13].

\section{Create a disaster manual of policies and procedures}

Each hospital should develop a predesigned series of protocols and operating procedures for pregnant patients in case of a pandemic, mass casualty incident, or natural disaster [13]. These documents should be adaptable to disease specifics and updated promptly as new disease management recommendations emerge. The components of documents should include information in Table 1. Examples of disaster response manuals can be found via medical society websites, such as the Society of Critical Care Medicine [14]. These documents highlight separate from usual processes for patients during a pandemic and are tiered based upon hospital resources and capacity to provide patient care. The three categories that are useful to include: conventional, contingency, and crisis care $[15,16]$.

Conventional care refers to the hospital capacity to have an adequate staff, resources, room availability, and supplies to care for patients. There may be altered processes based on the population management of the pandemic, such as limitation of elective procedures, screening, protective equipment, and altered patient flow. In this setting, patients are cared for within usual patient care environments. In a surge of patients beyond this accommodation, this may initiate the contingency care where there are indications that resources may be limited in the near future. This may require expanding to alternative patient care settings, transferring patients to other facilities, consideration for rationing resources, and/or securing additional resources. All patients are still adequately cared for within the hospital; however,
Table 1: Components of a disaster manual made specific to hospital.

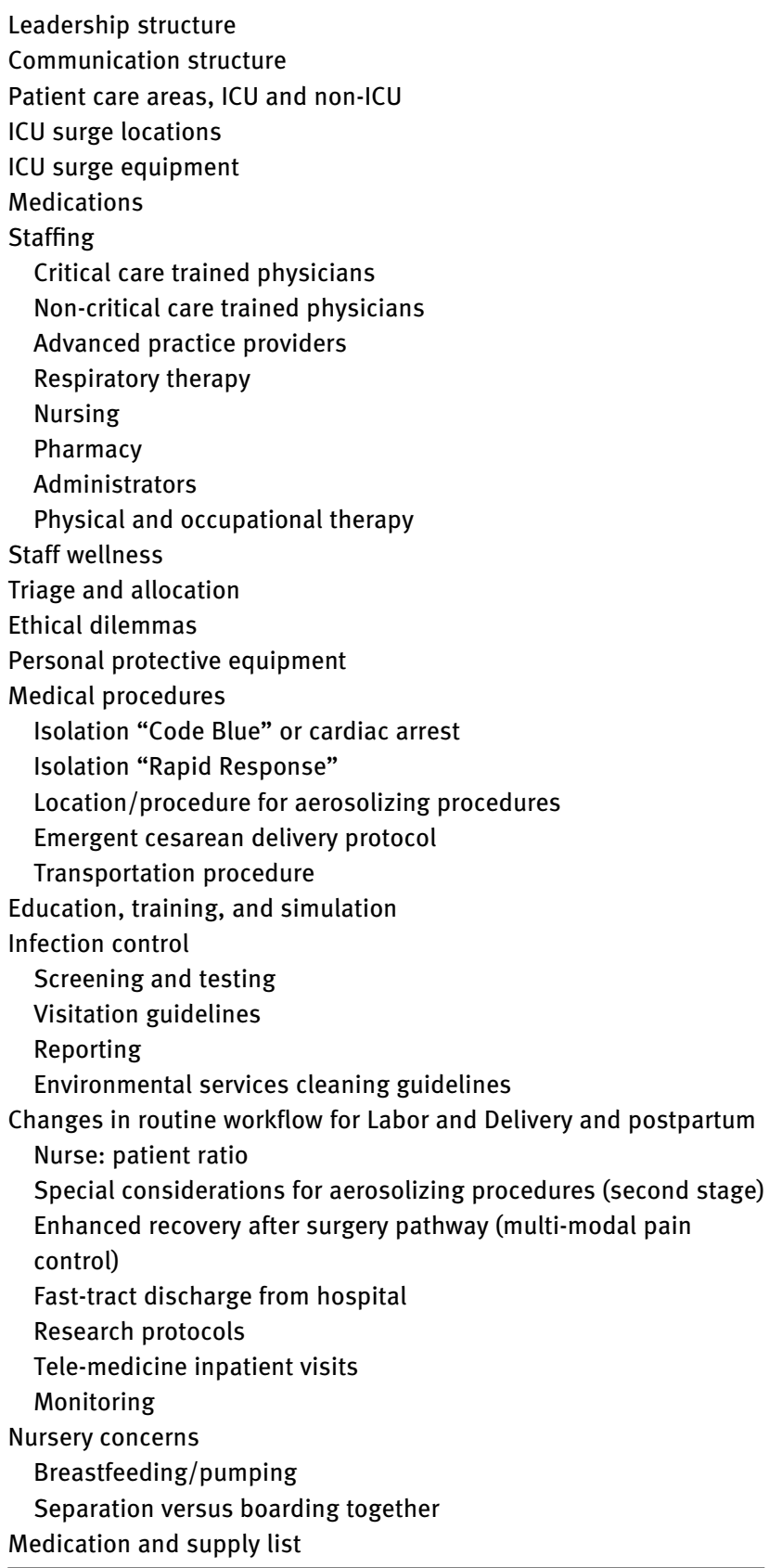

initiation of "triage assessment scoring" and creation of the triage committee will begin in case of surge progression to exceed beyond the hospital's capacity. There may be a conceptual shift from the "first come-first served" model to a "triage" model, with ethical drivers for the "greatest good for the greatest number of people." Crisis care is the final stage where the volume of patient needs exceed that of the hospital resources, and treatment allocation is in effect. During this stage, there may be consideration for universal 
"Do not resuscitate" in case of a cardiac arrest, especially if likelihood of survival is poor in the setting of the pandemic. Triage allocation committees are formed to guide clinicians on who should receive life sustaining treatment. These concepts need to be fully considered within the context of the community the hospital serves for ethical and legal support (Figure 1) [15, 16].

A diverse and multidisciplinary team comprised of Critical Care physicians, Maternal Fetal Medicine Specialists, head nurses, hospital administrators, community leaders, ethicists, legal authorities, and government officials should create these processes and protocols. A goal of this team is to provide appropriate inclusivity of the community it serves and to deliver high quality of care to serve the most patient-life years [13]. Pregnancy will offer an additional challenge, as the patients are relatively young, have differing therapeutic goals based on maternal physiology, and need to account for fetal implications of therapy.

\section{Communication with hospital system/region}

During a crisis such as a pandemic, it is likely that multiple hospitals will be managing their own surge of patients. General hospitals without high-volume obstetric services may divert pregnant patients to a specialized obstetric hospital to utilize their own Labor and Delivery (L\&D) bedspace for sick adult patients. In this situation, the regional obstetric hospital will need to accommodate a larger volume of routine obstetrics as well as critically ill obstetric patients. A hospital region may designate a "Regional Care Committee" that has up-to-date knowledge of patient acuity, resources, and patient volume at each affiliate hospital. This committee's lead (like a "Care Czar") will communicate to the Incident Commanders (IC), commonly an Intensivist, of each hospital in a Health Insurance Portability and Accountability Act (HIPAA) compliant platform in the USA with real-time updates. The Regional Care Committee will work with the legislature, medical supply chain, and hospital administrators to ensure appropriate distribution of patients and resources amongst the hospitals. They also should be responsible for updating emergency transport personnel on where to take patients based on specific complaints or pregnancy. Regional Care Committees may also advise the state leadership on distribution of essential materials, provider protections for civil/legal lawsuits, and create a command structure for reporting hospital resource needs (Figure 2).

The IC of the obstetric hospital should have a format to review the hospital census, patient acuity, supply chain issues, medication shortages, and needs for personal protective equipment (PPE) and personnel to report to the Regional Care Committee in real time. This level of communication should be indoctrinated at least daily while in a hospital contingency or crisis capacity of care. The IC should also be communicating with hospital administration for cancellation of elective procedures, conservation of medications and equipment, division of infected versus non-infected patients, and personnel
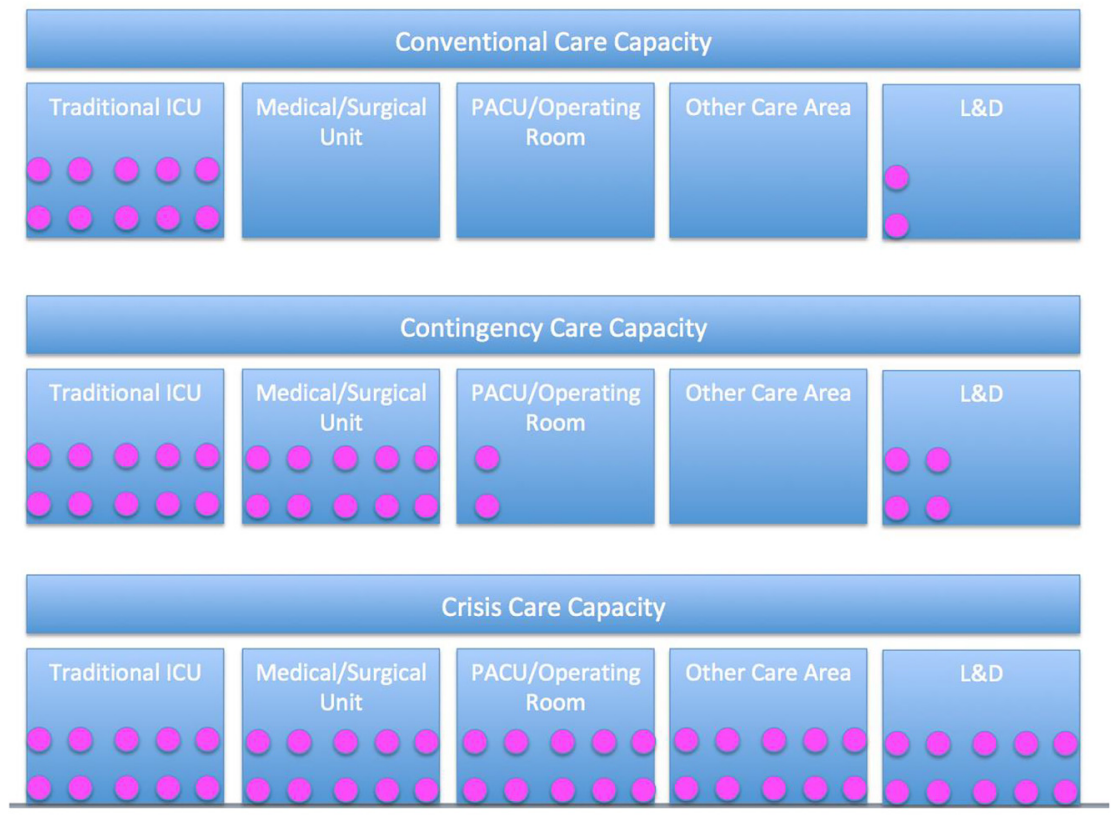

Represents an ICU patient
Figure 1: Example of a surge capacity at a hospital, utilizing L\&D and other care areas as last resort for critically ill patients. 
support. It should be noted that many obstetric complaints in addition to labor are not elective. Developing processes for pregnant patients who are low versus high risk for acquiring the illness shall designate particular hospital spaces as to prevent the spread of infection within the hospital. Open discussion of these protocols with the public will ensure confidence and demonstrate patient safety within the hospital, promoting community trust in health care.

\section{Medical algorithm alteration}

Specific alterations on policies for obstetric patients should be considered during a pandemic, such as in postpartum hemorrhage (PPH), rapid response, cardiac arrest, and emergency cesarean deliveries. Blood banks may have diminished blood product supply due to restrictive donation policies. During a "regional blood restriction," consideration for earlier intervention with uterotonic medications and/or anti-fibrinolytics should be considered. Permissive hypotension and transfusion of fewer units may also be helpful in conserving blood supply in appropriate patients.

Many protocol modifications are created with the primary emphasis to protect medical staff, particularly in emergencies. The American Heart Association announced
Advanced Cardiac Life Support modifications to COVID-19 infected patients to protect responders during a cardiac arrest. These include donning appropriate PPE, limiting to essential personnel, placing a mask on the patient prior to initiating cardiopulmonary resuscitation (CPR), providing "hands-only CPR", avoiding bag-valve-mask ventilation, early intubation with video laryngoscopy, attaching an HEPA and viral filters, and consider back compressions in prone patients that arrest [17]. These principles also translate into rapid response of a patient, following similar algorithms. Creating an addendum to existing protocols, or creating separate protocols altogether, will identify these processes and is prudent health care provider (HCP) protection. We created addendums to existing protocols as "Isolation Code Blue" and "Isolation Rapid Response" triggers, for providers to utilize these extra precautions when responding to a patient.

Another important consideration is for the emergency transfer of a patient to the operating room for a cesarean delivery. Precautions must be made to prevent the spread of infection to HCP and other patients whilst providing standard of care for the infected patient. Developing a standardized protocol should include donning and doffing of personal protective equipment (PPE), designating roles to specific providers, physical pathway to a designated operating room, anesthesia considerations, and reduction to only essential staff to be involved for the

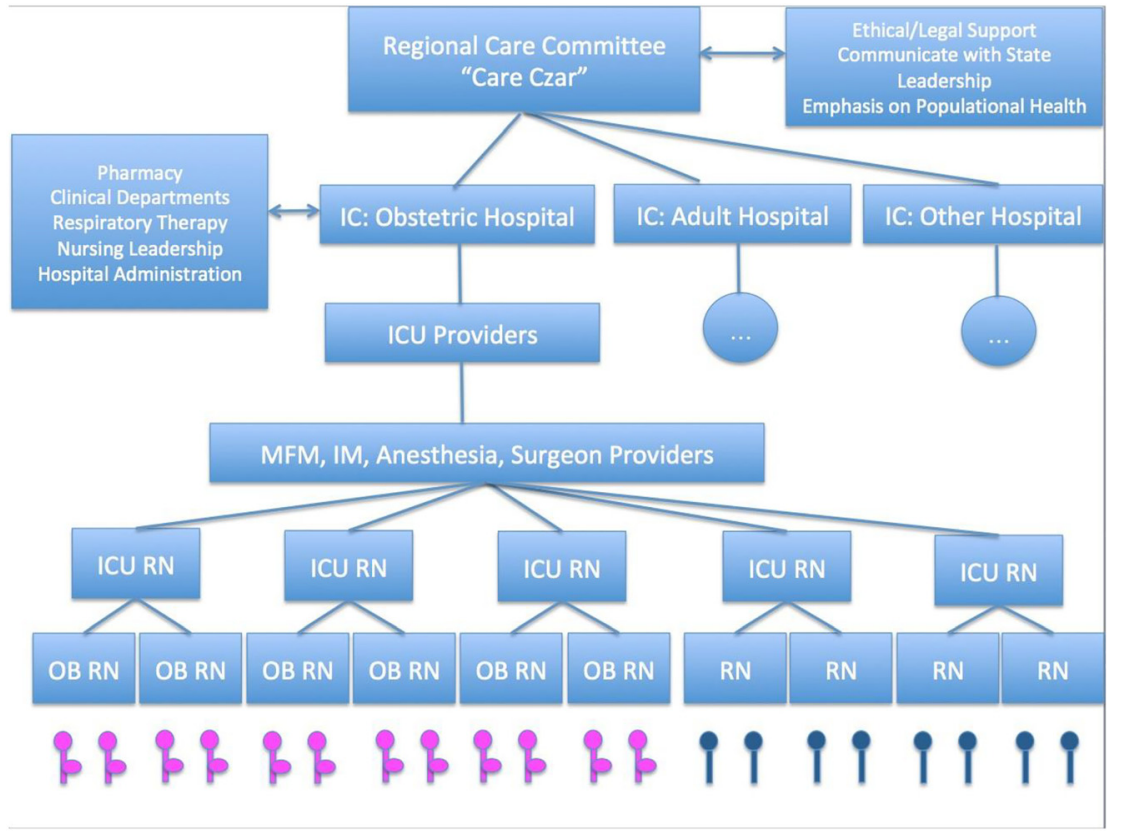

IC: incident commander, ICU: intensive care unit, MFM: maternal fetal medicine, IM: internal medicine, RN registered nurse, OB: obstetric. $\$$ Critically ill pregnant patient. $\uparrow_{\text {Critically ill }}$ postpartum/non-pregnant patient.
Figure 2: Organizational chart describing the structure of care in a region to its obstetric hospital, including a hospital's structure for patient care. 
delivery. Placing a fetal scalp electrode for a fetal deceleration may reduce haste during this process if the fetal status improves during transport. Providing multiple simulations (see Education, Training, and Simulation) will aid in perfecting the process in addition to educating the staff (Appendices 1 and 2).

Discussions regarding universal screening of patients and/or visitors should be considered based on the incidence and prevalence of the disease in the community. Advocates for universal screening tout less usage of PPE, improved provider safety, and assessment of true incidence of the disease in the community. However, this may be an expensive or burdensome process, depending on the disease and the screening test needed. It is important to know the positive and negative predictive values of the test, and that this test is in "a point of time" where the patient may not currently be shedding the virus. Infection control personnel can help with these recommendations.

\section{Surge capacity - patient care areas}

A surge of hospitalized and critically ill patients may happen precipitously. Predetermined algorithms for patient distribution within the hospital need to have several considerations: 1) negative pressure isolation rooms or units, 2) staff availability, 3) procedural concerns (i.e., patient in active labor to be cared for on L\&D), 4) availability of equipment, or 5) physical separation of infected versus non-infected patients (i.e. place all patients on a "COVID Unit," regardless of level of care), or 6) transfer patient to a "COVID" facility. Each hospital will need to individualize the approach to this process as it balances physical space, personnel, monitoring equipment, and accessibility to medications. In general, critical care provisions should go to the patient, and location of services should be where the highest acuity problem can be most easily treated. If the critical care needs exceed the risk of delivery, the patient may be considered to go to the ICU and have delivery equipment immediately available. If the patient is likely to deliver shortly, then ICU monitoring and treatment may be best provided on L\&D. For this reason, we retrofitted several $L \& D$ rooms to allow for ICU monitoring and, conversely, have fetal monitoring capabilities in several ICU rooms. Additionally, having areas dedicated to high-risk procedures is important in infections that transmit via aerosolizing procedures. Having a dedicated "intubation and bronchoscopy" room may be useful to limit disease transmission.

In a conventional care capacity, patients are placed where they usually would be dependent on their level of care. As the hospital approaches contingency or crisis care capacity, patients may be distributed to locations in the hospital that are unconventional $[13,18]$. This should be done in a structured and tiered approach, to expand care areas as needed (Figure 1). These areas should have appropriate monitoring modalities (fetal heart rate, cardiac monitor, and pulse oximetry), resuscitation stations for babies, areas for procedures, and other therapeutic interventions (intravenous access, oxygen, etc.,). The expansion areas can include ICU overflow areas, "step down units," L\&D, older and currently unused clinical areas, post-anesthesia care units (PACU), operating rooms, and non-clinical areas (i.e., auditorium). Patient characteristics to be admitted to each unit should be pre-defined and correlate to the severity of illness.

We currently are accredited for a three bed Adult Intensive Care Unit with two overflow ICU beds, which would be insufficient for a large surge of patients requiring critical care. Working with our administration, we found 40 possible individual bed locations that had appropriate monitoring capability, oxygen and air connections, generator backup power, and workstations for staff and providers. Some of these areas had been converted to administrative services, but with minimal interventions could be readied for patient care. Direction of airflow from positive pressure and negative pressure environments also determined candidacy for location of care services.

\section{Surge capacity - personnel}

Trained HCP's in critical care medicine is usually the rate-limiting step for treating a large volume of critically ill patients and a tiered staffing plan may need to be implemented to effectively treat the most patients (Figure 3) [13]. Although is important to keep providers within their scope of practice, intensivists can monitor and lead non-intensivist providers for critical care. There are some treatment modalities that define critical care (i.e., vasoactive medications and mechanical ventilation); however, there are multiple other aspects of care that can be managed by providers from other specialties. Having a multidisciplinary team divide tasks to treat the critically ill patient with constant communication with the intensivist may accommodate the large volume of patients during a surge.

Example Scenario: You have one intensivist per shift and 30 critical care patients. The intensivist can physically locate in the unit with the sickest patients with scheduled rounds with other providers. The surgeon on-call can perform procedures such as placement of central and 
arterial access catheters, tube thoracostomies, and tracheostomies. Anesthesia providers can aid in intubations, sedation, and airway management. Maternal Fetal Medicine Specialists (perinatologist) can manage the obstetric component including recommendations for delivery, antenatal steroids, fetal monitoring, and patient positioning. Internal medicine can aid in antibiotic selection, nutritional supplementation, and electrolyte supplementation. This multi-disciplinary team will direct ICU nurses on patient care. If the ICU nurse to patient ratio exceeds their capacity to carry out patient orders (i.e., usually defined as greater than one nurse to two patients), then utilizing obstetric and floor nurses to perform patient care within their scope of practice can aid the ICU nurse on non-ICU-related tasks. Although it is not ideal, this is a proposed model to collaboratively treat a large surge of ICU patients. Especially with nursing, it is important to keep HCP's strictly within their scope of practice due to competency training and medico-legal concerns. Non-ICU trained nurses should not be titrating vasopressors. Having trainees such as residents or fellows can be helpful in placing orders and providing documentation during this process, as well as with direct patient care.

In this model, it is important to have a clearly defined command structure, such as in listed in Figure 2, to maximize ICU treatment ability. Defined roles and responsibilities for each provider in addition to a "patient checklist" will ensure that each patient receives comprehensive ICU care. Non-clinical personnel should not provide clinical care. Having legislature supported documentation in a "state of emergency", that practitioners have additional medico-legal protection from civil and criminal lawsuits will support providers to treat patients in these extraordinary circumstances.[13] Additionally, turning off "nuisance" alarms, streamlining documentation with note templates, and providing accessible hand-washing stations will make patient care more efficient. There are many ICU specific care modifications for COVID-19, but are constantly changing and beyond the scope of this article [18].

\section{Education, training, and simulation}

Providing effective education for HCP's is the most important. Knowledge empowers the team to take a safe approach to patient care, ensures confidence, and boosts morale [19]. This was clearly evident in our hospital when physicians in leadership positions scheduled unit-based educational updates, question-answer sessions, and most importantly, simulation training. Anecdotally, multiple nurses and respiratory technicians declared that they became "ready" to care for infected COVID-19 patients in this pandemic when they had previously expressed feelings of trepidation.

PPE donning/doffing and hand-washing are cornerstones to promote individual safety, with trained observers to determine competency in the task. Unit orientation for new devices, such as a video laryngoscopes or disposable

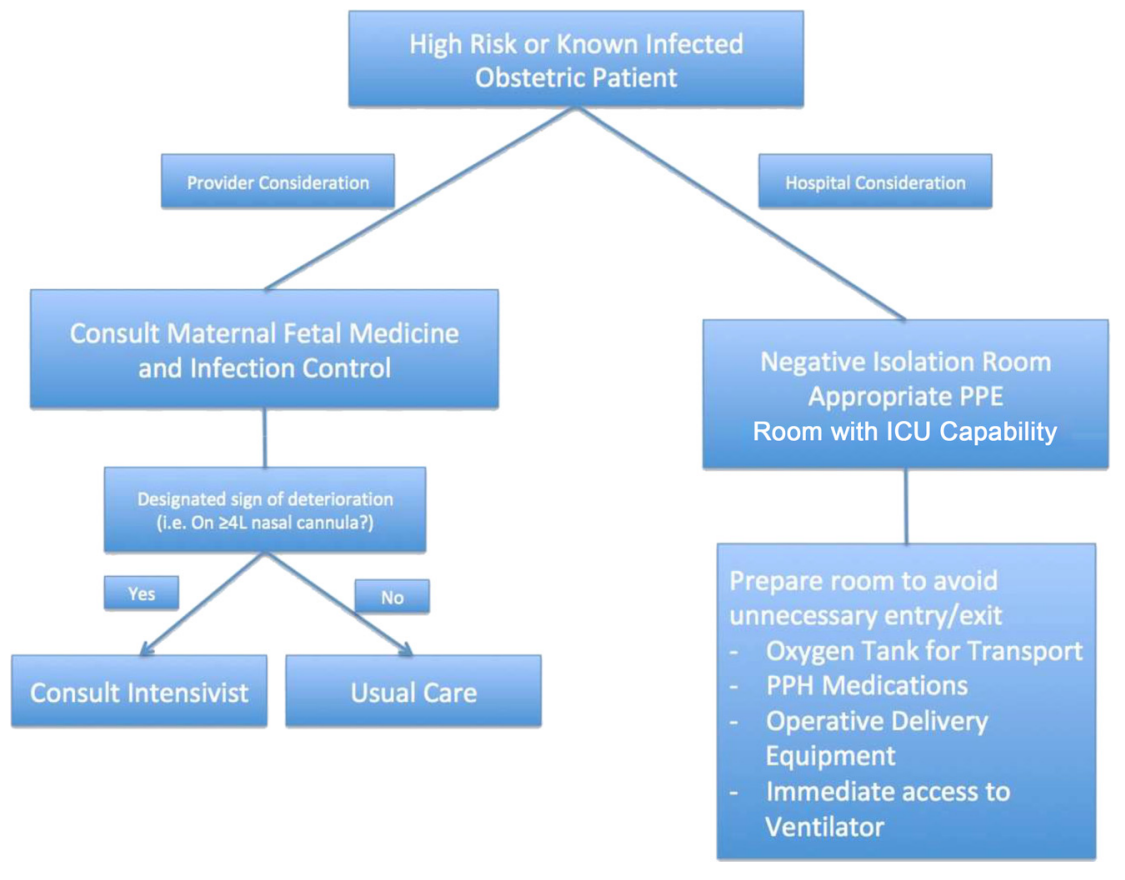

Figure 3: Algorithm for initial management of the patient. 
bronchoscopes, their uses and specifications, will ensure adequate usage of the new equipment. Ensuring that HCP's will provide care within their scope of practice and have immediate clinical support also boosts confidence in treating patients.

Development of new protocols or practice guidelines from one specialty's perspective can be deleterious to patient care. Involving colleagues in a multi-disciplinary team approach will provide a myriad of different approaches to patient care. This team approach should be used to develop practice guidelines, followed by educating staff, and performing "walk-throughs." Physically performing tasks on the unit, the team can determine what aspects of the process are not feasible (i.e., the bed does not fit in a particular set of elevators!) and revise the protocol prior to simulation testing. After the team determines a process as adequate with a walkthrough, testing it with a multi-disciplinary team that was not directly involved in the protocol's inception generates more feedback to be incorporated into the protocol. Once the protocol is (mostly) seamless and practical, then finalization of the protocol and widespread unit education should begin (Figure 4). We also created online narrated lectures for HCP's to review at their leisure and pace (Appendix 1). Tracking attendance for simulations on the units for both day and night shift providers will ensure that staff has been adequately trained for the pandemic. If time has elapsed from the onset of training and a new surge, providing "just in time" simulation training will boost knowledge and improve outcomes just prior to treating patients [20]. As often termed, "culture eats strategy for lunch," efforts to indoctrinate the practice guideline into a hospital unit's culture will mark its successful implementation. Leadership that is receptive to feedback, and make adaptations due to input, will have higher unit compliance. Lastly, providing staff with emotional support and wellness activities will also contribute to a good working environment during stressful events.

\section{Hospital resources}

Ensuring adequate hospital resources may also be a ratelimiting step to adequate patient treatment. The United States Food and Drug Administration indications for medical devices, medications, and nutritional supplements should be respected and utilized as directed. Pharmacy directors should provide timely and interval communications to hospital leaders regarding medication shortages and reasonable alternatives. Conservation efforts by sending adequate and not superfluous doses of medications to patients will aid in maximizing medication utility. Keeping an adequate par and list of intravenous pumps, tubing, invasive/non-invasive monitoring devices, and other supplies will prompt when to restock supplies. A full list of utilized medications and supplies, including mechanical ventilators, should be checked at least daily.

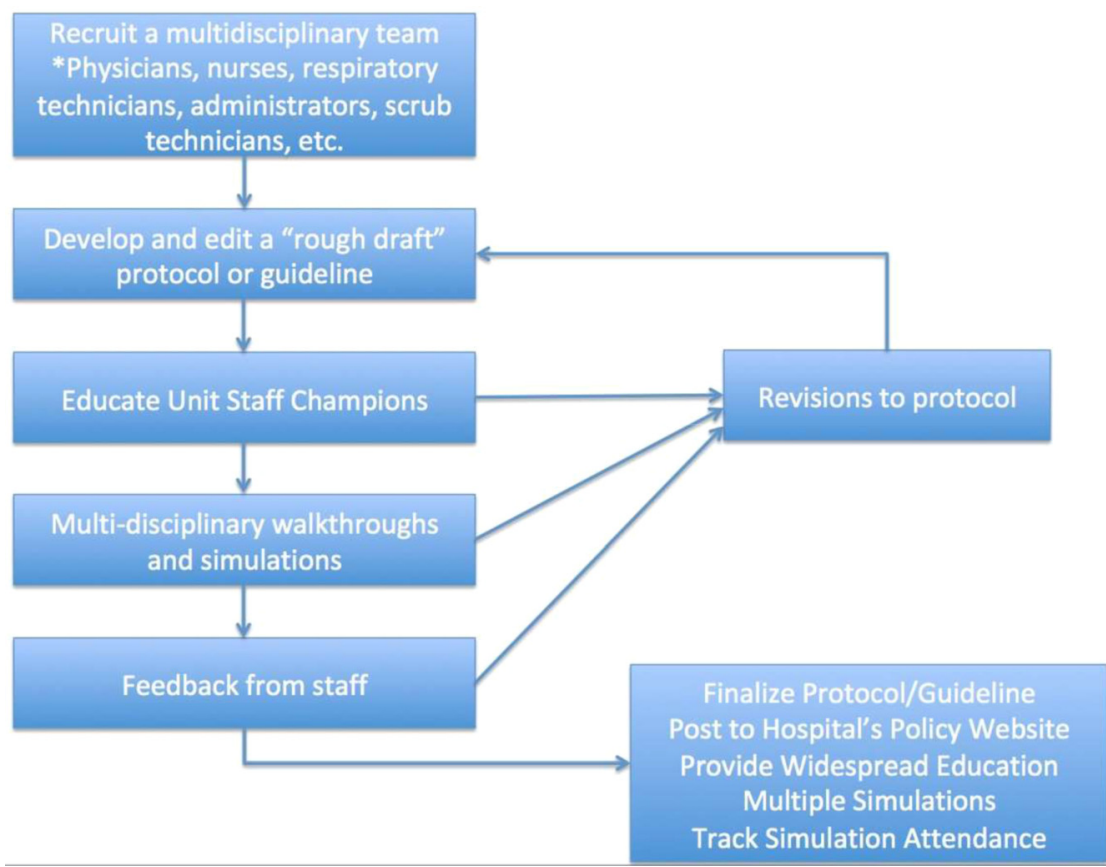

Figure 4: Approach to writing a new practice protocol or guideline for a pandemic, natural disaster, or mass casualty incident. 


\section{Triage and allocation of resources}

The concept of "triage" is one that is used routinely in medicine to allocate resources in a timely manner to meet needs of individual patients. However, the concept transcends from its conventional daily use to crisis allocation when patient volume exceeds hospital resources. Clinicians may need to choose who may, and who may not, utilize hospital resources [21]. We developed a comprehensive scoring tool evaluating intact survivability of patients who contract a disease, incorporating COVID-19 disease specifics into the calculator. After honoring advanced health care directives and during a contingency or crisis care capacity, scores would be calculated daily for all ICU patients to determine utilization of scarce hospital resources [6, 22]. Focusing on principles of "saving the most life-years" in an ethical standard is challenging and requires administrative and governmental support [6]. Some scoring tools include pregnancy as an "extra point" to consider the life of the fetus, as saving the "most lives" and the greatest number of potential years of life [15, 22, 23]. A multidisciplinary committee that comprised of physicians, hospital administrators, community leaders, ethicists, legal authorities, and legislatures created these documents that needed to be devoid of racial/ethnic, gender, or social status biases [13]. A fair and transparent representation of this process, with an appeals mechanism, was by far the most challenging aspect of this pandemic to write.

\section{Conclusions}

The COVID-19 pandemic encouraged our hospital for emergency preparedness by developing policies and procedures, education, and contingency plans for a surge of infected, critically ill obstetric patients. There is a plethora of information pertaining to the therapy and treatment of COVID-19, but few manuals on how to prepare a specialized obstetric hospital for such a pandemic. While we dissect out updated principles of disease and pandemic management, we created this document to guide other hospitals to create their own personalized approach to manage a pandemic surge.

Research funding: None declared.

Author contributions: All authors have accepted responsibility for the entire content of this manuscript and approved its submission.

Competing interests: Authors state no conflict of interest.

\section{Appendix 1}

Example of online lecture for treatment team on emergency cesarean delivery.

https://drive.google.com/file/d/1AOUsYVc7VZq8Nlbf

B1pNzk3zxfqh1yaQ/view?usp=sharing

\section{Appendix 2}

Sample algorithm for an emergent cesarean delivery, role of the attending physician on-call.

Emergent cesarean delivery process-attending physician

- Enter Anteroom

- Don PPE (N95, Face shield, Surgical cap/bouffant, gown, gloves, shoe covers)

- Assess patient

Consider Fetal Scalp Electrode

Perform resuscitative measures

Early call for cesarean delivery (don't wait 6 min on a decelerations-

THESE ARE SLOWER due to nursing process changes!)

- The intercom of the room will be on, so you can talk freely with the charge RN->notify them for "isolation emergent cesarean delivery"

- DOFF the booties, gown, and gloves in the anteroom. (Keep your head gear on) and WASH HANDS

- Go to OR-C and scrub for surgery and stand in the corner and wait

- MD Attending A will be assisted by MD Attending B. Please call in MFM on call for in-house backup.

- Complete the cesarean delivery

- DOFF booties, gown and gloves in the OR (Wash hands).

- DOFF face shield and hat in OR core (Wash hands).

- DOFF N95 in triage area (Wash hands).

- Consider retrieving new scrubs and shower.

The nurses will be designated to the COVID patients and will have specific roles. They are not to move the patient until the OR and anesthesiologist is ready. The OR doors will NOT HAVE POWER, meaning, you have to pull them open. There will be a hallway monitor to clear the pathway.

\section{References}

1. CDC COVID-19 Response Team. Severe outcomes among patients with coronavirus disease 2019 (COVID-19) - United States February 12-March 16, 2020. MMWR Morb Mortal Wkly Rep 2020;69:343-6.

2. Sahu K, Mishra AK, Lal A. COVID-2019: update on epidemiology, disease spread and management. Monaldi Arch Chest Dis 2020; 90. https://doi.org/10.4081/monaldi.2020.1292 [published ahead of print 2020 Apr 16]. 
3. Wu ZMJ. Characteristics of and important lessons from the coronavirus disease 2019 (COVID-19) outbreak in China: summary of a report of 72,314 cases from the Chinese center for disease control and prevention. J Am Med Assoc 2020;323:1239-92.

4. Centers for Disease Control and Prevention. Coronavirus disease 2019 (COVID-19) cases in US. https://www.cdc.gov/coronavirus/ 2019-ncov/cases-updates/cases-in-us.html [Accessed 10 Jun 2020].

5. Li X, Ma X. Acute respiratory failure in COVID-19: is it "typical" ARDS?. Crit Care 2020;24:198.

6. Sprung CL, Joynt GM, Christian MD, Truog RD, Rello J, Nates JL. Adult ICU triage during the coronavirus disease 2019 pandemic: who will live and who will die? recommendations to improve survival. Crit Care Med 2020. https://doi.org/10.1097/CCM. 0000000000004410 [In press].

7. Dashraath P, Wong JLJ, Lim MXK, Lim LM, Li S, Biswas A, et al. Coronavirus disease 2019 (COVID-19) pandemic and pregnancy. Am J Obstet Gynecol 2020;222:521-31.

8. Wong SF, Chow KM, Leung TN, Ng WF, Ng TK, Shek CC, et al. Pregnancy and perinatal outcomes of women with severe acute respiratory syndrome. Am J Obstet Gynecol 2004;191:292-7.

9. Alfaraj SH, Al-Tawfiq JA, Memish ZA. Middle East respiratory syndrome coronavirus (MERS-CoV) infection during pregnancy: report of two cases \& review of the literature. J Microbiol Immunol Infect 2019;52:501-3.

10. Schwartz DA, Graham AL. Potential maternal and infant outcomes from (Wuhan) Coronavirus 2019-nCoV infecting pregnant women: lessons from SARS, MERS, and other human coronavirus infections. Viruses 2020;12:194-210.

11. Sjödin H, Wilder-Smith A, Osman S, Farooq Z, Rocklöv J. Only strict quarantine measures can curb the coronavirus disease (COVID-19) outbreak in Italy, 2020. Euro Surveill 2020;25:200-6.

12. Coronavirus Disease. (COVID-19); 2019. Available from: https:// www.cdc.gov/coronavirus/2019-ncov/cases-updates/cases-inus.html.

13. Sprung CL, Zimmerman JL, Christian MD, Joynt GM, Hick JL, Taylor B, et al. Recommendations for intensive care unit and hospital preparations for an influenza epidemic or mass disaster: summary report of the European society of intensive care medicine's task force for intensive care unit triage during an influenza epidemic or mass disaster. Intensive Care Med 2010; 36:428-43.

14. Farmer JCWR, Baldisseri MR. Preparing your ICU for disaster response. Soc Crit Care Med 2012:270.

15. Daugherty Biddison EL, Faden R, Gwon HS, Mareiniss DP, Regenberg AC, Schoch-Spana M, et al. Too many patients a framework to guide statewide allocation of scarce mechanical ventilation during disasters. Chest 2019;155:848-54.

16. Hick JLBJA, Kelen G.D. Refining surge capacity: conventional, contingency, and crisis capacity. Disaster Med Public Health Prep 2009;3:S59-67.

17. Edelson DP, Sasson C, Chan PS, Atkins DL, Aziz K, Becker LB, et al. Interim guidance for basic and advanced life support in adults, children, and neonates with suspected or confirmed COVID-19: from the emergency cardiovascular care committee and get with the guidelines-resuscitation adult and pediatric task forces of the American heart association. Circulation 2020; 141:e933-43. https://doi.org/10.1161/circulationaha.120. 047463 [Jun 23 Epub 2020 Apr 9].

18. Griffin KMKM, Ivascu NS, Lief L. Hospital preparedness for COVID19: a practical guide from a critical care perspective. Am J Respir Crit Care Med 2020;201:1337-44.

19. Devereaux A, Christian MD, Dichter JR, Geiling JA, Rubinson L. Summary of suggestions from the task force for mass critical care summit. January 26-27 2007. Chest 2008; 133:1S-7S.https://doi.org/10.1378/chest.08-0649.

20. Aggarwal R. Just-in-time simulation-based training. BMJ Qual Saf 2017;26:866-8.

21. Christian MD. Triage. Crit Care Clin 2019;35:575-89.

22. Maves RC, Downar J, Dichter JR, Hick JL, Devereaux A, Geiling JA, et al. Triage of scarce critical care resources in COVID-19 an implementation guide for regional allocation: an expert panel report of the task force for mass critical care and the American college of chest physicians, Chest. 2020 Apr 11:S0012-3692(20) 30691-7. Online ahead of printreport.

23. Emanuel EJ, Persad G, Upshur R, Thome B, Parker M, Glickman A, et al. Fair allocation of scarce medical resources in the time of Covid-19. N Engl J Med 2020;382:2049-55. 